\title{
JOINTLY OPTIMIZED ADAPTIVE RECURSIVE IDENTIFIER
}

\author{
E. A. SOLEIT
}

\section{ABSTRACT}

A unimodal stable adaptive recursive filter is introduced. The forward and the backward coefficients are jointly optimized such that two different performance indices are minimized. The performance criteria are quadratic functions of the filter coefficients. Moreover, the filter stability is always hyperstable during the adaptation process. This filter has less complexity than the direct form structure.

\section{INTRODUCTION}

The applications of the adaptive recursive digital filter (ARDF) in system identification, communication and control systems are attractive. This is due to the fact that the ARDF can match a physical system of poles and zeros with fewer number of coefficients than the adaptive transversal filter. Moreover, the recursive filter exhibits better frequency and dynamic responses over the transversal one $[1,2]$. However, the adaptive recursive filter suffers from tendency to instability and the multimodality of the performance criterion [1-4].

In this paper a proposed scheme of a unimodal stable adaptive recursive filter is presented. The forward and the backward filter coefficients are jointly optimized such that two different performance criteria are minimized. The forward performance criterion is the mean square error while the backward one is the mean square of the error function. The error function is derived from the filter error signal via an average processing. This scheme is always hyperstable during the adaptation process [5-7].

This paper has seven sections. Section two explains the canonical implementation of the recursive filter. The unimodal performance criteria are analyzed in section three while the adaptation algorithm is illustrated in section four. Section five analyses the stability of the proposed scheme. Section six presents the evaluation of the proposed algorithm through the computer simulation. Conclusions of the whole paper are given in section seven. 


\section{THE CANONI CAL STRUCTURE}

Generally, the recursive filter is described by the $\mathrm{M}^{\text {th }}$ order difference equation which is given by:

$$
y_{k}=\sum_{i=0}^{N} a_{i, k} x_{k-i}+\sum_{j=1}^{M} b_{j, k} y_{k-j}
$$

where $\left\{a_{i, k}\right\}$ and $\left\{b_{j, k}\right\}$ are the forward and the backward coefficients respectively. Also, $\left\{x_{k-i}\right\}$ and $\left\{y_{k-j}\right\}$ are the input and the output observations respectively. Furthermore, the filter structure can be expressed in the $\mathrm{z}$ domain as:

$Y(z)=\frac{\sum_{i=0}^{N} a_{i, k} z^{-i}}{1-\sum_{j=1}^{M} b_{j, k} z^{-j}} X(z)$

One can rearrange eq. (2) to take the form :

$$
Y(z)=\sum_{i=0}^{N} a_{i, k} z^{-i} W(z)
$$

where $W(z)$ is the output of an autoregressive processor that is defined by:

$$
W(z)=\frac{X(z)}{1-\sum_{j=1}^{M} b_{j, k} z^{-j}}
$$

It is obvious that the canonical form of the recursive filter is well described by equations (3) and (4).

\section{THE PERFORMANCE CRITERIA}

The mean square error criterion is usually used in the adaptive filtering techniques. The output error signal is defined as: 


$$
\varepsilon_{\mathrm{k}}=\mathrm{d}_{\mathrm{k}}-\mathrm{y}_{\mathrm{k}}
$$

where $d_{k}$ is the desired response and $y_{k}$ is the filter output. The filter output in eq. (3) can be written in time representation as:

$$
y_{k}=\sum_{i=0}^{N} a_{i, k} w_{k-i}
$$

Substituting eq. (6) in eq. (5) yields:

$$
\varepsilon_{k}=d_{k}-\sum_{i=0}^{N} a_{i, k} w_{k-i}
$$

Taking the expectation of the error square, the mean square performance criterion, $\xi_{k}$ is defined as [2]:

$$
\xi_{k}=E\left[\varepsilon_{k}^{2}\right]=E\left[d_{k}^{2}\right]-2 A_{k}^{T} P_{d}+A_{k}^{T} R_{k} A_{k}
$$

where $A_{k}$ is the forward coefficient vector which is defined by :

$$
A_{k}^{T}=\left[a_{0, k} a_{1, k} \cdots a_{N, k}\right]
$$

and $\mathrm{P}_{\mathrm{d}}$ is the cross-correlation vector that is written as:

$$
P_{d}=E\left[d_{k} W_{k}\right]
$$

where the observation vector $\mathrm{w}_{k}$ is defined as:

$$
\mathrm{w}_{\mathrm{k}}=\left[\begin{array}{llll}
\mathrm{w}_{\mathrm{k}} & \mathrm{w}_{\mathrm{k}-1} & \cdots & \ldots \\
\mathrm{w}_{\mathrm{k}-\mathrm{N}}
\end{array}\right]
$$

The autocorrelation matrix, $\mathrm{R}_{\mathrm{k}}$ is defined by:

$$
\mathrm{R}_{\mathrm{k}}=\mathrm{E}\left[\mathrm{W}_{\mathrm{k}} \mathrm{W}_{\mathrm{k}}^{\mathrm{T}}\right]
$$

It is clear that $\xi_{\mathrm{k}}$ is a quadratic function of the forward coefficient vector, $A_{k}$. Then, differentiating $\xi_{k}$ with respect to $A_{k}$ and equating the derivative to zero yields:

$$
A_{k}=R_{k}^{-1} P_{d}
$$


Equation (13) is known as the filter normal equation [1-3]. Substituting eq. (1) in eq. (6) and rearrange the resulting formula yields:

$$
\varepsilon_{k}=d_{k}-\sum_{i=0}^{N} a_{i, k} x_{k-i}-\sum_{j=1}^{M} b_{j, k} d_{k-j}+\sum_{j=1}^{M} b_{j, k}\left(d_{k-j}-y_{k-j}\right)
$$

One can write eq. (14) in the form of:

$$
\rho_{\mathrm{k}}=r_{\mathrm{k}}-\mathrm{B}_{\mathrm{k}}^{\mathrm{T}} \mathrm{D}_{\mathrm{k}}
$$

where $\rho_{k}$ is defined as the error function and it is defined by :

$$
p_{k}=\varepsilon_{k}-\sum_{j=1}^{M} b_{j, k} \varepsilon_{k-j}
$$

and $r_{\mathrm{k}}$ is given as :

$$
r_{k}=d_{k}-\sum_{i=0}^{N} a_{i, k} x_{k-i}
$$

$\mathrm{B}_{\mathrm{k}}$ is the backward coefficient vector that is expressed as:

$$
\mathrm{B}_{\mathrm{k}}^{\mathrm{T}}=\left[\mathrm{b}_{1, \mathrm{k}} \mathrm{b}_{2, \mathrm{k}} \cdots \mathrm{b}_{\mathrm{M}, \mathrm{k}}\right]
$$

Moreover, $\mathrm{D}_{\mathrm{k}}$ is known as the observation vector of the previous desired responses which is expressed by :

$$
D_{k}^{T}=\left[d_{k-1} d_{k-2} \cdots d_{k-M}\right]
$$

Consequently, the mean square of the error function is described by:

$$
\Gamma_{\mathrm{k}}=\mathrm{E}\left[\mathrm{r}_{\mathrm{k}}^{2}\right]-2 \mathrm{~B}_{\mathrm{k}}^{\mathrm{T}} \mathrm{P}_{\mathrm{r}}+\mathrm{B}_{\mathrm{k}}^{\mathrm{T}} \mathrm{H} \mathrm{B}_{\mathrm{k}}
$$

where the autocorrelation matrix, $\mathrm{H}$ is defined as :

$$
\mathrm{H}=\mathrm{E}\left[\mathrm{D}_{\mathrm{k}} \mathrm{D}_{\mathrm{k}}^{\mathrm{T}}\right]
$$

and $\mathrm{P}_{r}$ is the cross-correlation vector that is given by :

$$
\mathrm{P}_{\mathrm{r}}=\mathrm{E}\left[\mathrm{r}_{\mathrm{k}} \mathrm{D}_{\mathrm{k}}\right]
$$

Differentiating eq. (20) with respect to the backward coefficients and equating the derivative to zero yields the normal equation for the backward coefficients: 


$$
\mathrm{B}_{\mathrm{k}}=\mathrm{H}^{-1} \mathrm{P}_{\mathrm{r}}
$$

\section{THE ADAPTATION ALGORI THM}

The well known least mean square (LMS) adaptation algorithm is employed to update the forward and the backward coefficients $[1,2]$. The forward coefficients are updated such that the mean square error, $\xi_{\mathrm{k}}$ is minimized while, the backward coefficients are updated to minimize the mean square of the error function criterion, $\Gamma_{\mathrm{k}}$. The LMS adaptation algorithm for the forward coefficients can be expressed as:

$$
\begin{gathered}
a_{i, k+1}=a_{i, k}+2 \mu \varepsilon_{k} \frac{\partial \varepsilon_{k}}{\partial a_{i, k}} \\
i=0,1, \ldots \ldots \ldots N
\end{gathered}
$$

where $\mu$ is the step size that controls the adaptation speed and the stability of the adaptation algorithm. Substituting eq. (7) in eq. (24) the forward adaptation algorithm becomes

$$
a_{i, k+1}=a_{i, k}+2 \mu \varepsilon_{k} w_{k-i}
$$

Similarly, the backward coefficients are updated according to the following algorithm:

$$
\mathrm{b}_{\mathrm{j}, \mathrm{k}+1}=\mathrm{b}_{\mathrm{j}, \mathrm{k}}+2 \mu \rho_{\mathrm{k}} \frac{\partial \rho_{\mathrm{k}}}{\partial \mathrm{b}_{\mathrm{j}, \mathrm{k}}}
$$

Substituting eq. (15) in eq. (26) yields the LMS adaptation algorithm for the backward coefficients as:

$$
\begin{gathered}
b_{j, k+1}=b_{j, k}+2 \mu \rho_{k} d_{k-j} \\
j=1,2, \ldots \ldots \ldots M
\end{gathered}
$$

\section{THE FILTER STABILITY}

The stability of the canonical structure is ensured according to the hyperstability theorem $[6,7]$. Equation (14) can be represented diagrammatically by an unforced nonlinear feedback system as shown in Figure 1. The forward loop transfer function $G(z)$ is defined by: 


$$
G(z)=\frac{1}{1-\sum_{j=1}^{M} b_{j, k} z^{-j}}
$$

It is clear from eq. (16) that the error function, $\rho_{k}$ is generated from the error signal, $\varepsilon_{\mathrm{k}}$ via an average processor whose coefficients are identical to the filter backward coefficients. consequently the resultant forward loop transfer function will be unity which is always positive real for all $z$. Hence, the error function, $\rho_{k}$ tends to zero as $k$ tends to infinity and the filter stability is always ensured according to the hyperstability theorem [6,7].

\section{SIMULATION RESULTS}

The proposed canonical form of the adaptive recursive filter is demonstrated in Fig. 2. The performance of the proposed scheme is evaluated in the transient and the steady state modes when the filter is utilized in system modeling.

The model in this paper is represented by a second order transfer function as:

$$
H(z)=\frac{1-1.8 z^{-1}+1.1 z^{-2}}{1-1.2 z^{-1}+0.6 z^{-2}}
$$

The adaptive filter order is chosen to be identical to the model system. The input signal is chosen to be a white noise with a zero mean.

The transient response is described by the learning curve as illustrated in Fig. 3. It is clear that the filter converges to - $50 \mathrm{db}$ after 10000 iterations.

The steady state response is explained by the residual mean square error of the misalignment vector of the filter coefficients after convergence. The residual mean square error is $-200 \mathrm{~dB}$ after 30000 iterations. Moreover, the filter coefficients converge to the same values as those of the model. Table 1 demonstrates the steady state values of the filter coefficients.

Furthermore, it is found that the proposed filter has the same computation requirements ( $2 \mathrm{~N}+3 \mathrm{M}$ operations) as the SHARF filter that is presented by Larimore and the others in [6]. Moreover, the proposed algorithm requires a smaller memory storage than the SHARF algorithm. In addition to the precedings, the output mean square index, $\xi_{k}$ is a unimodal function and has a unique optimal solution while that of the SHARF one is a multimodal function which has local minima in addition to the global minimum solution. 


\section{CONCLUSI ONS}

The proposed canonical structure of the adaptive recursive filter has a unimodal error surfaces and it is always hyperstable which overcomes the main drawbacks of the direct form structure. The least mean square adaptation algorithm is derived to update the forward and the backward coefficients. The proposed algorithm can be implemented efficiently in real time with moderate complexity using the available digital signal processors.

\section{References}

[1] Treichler J. R., Johnson C. and Larimore M. G., " Theory and Design of Adaptive Filters", John Wiley \& Sons, New York, 1987.

[2] Widrow B. and Stearns S. D., "Adaptive Signal Processing", Prentice Hall, Inc, Englewod, 1985.

[3] Soleit E. A., "Adaptive Digital Filter Algorithms and Their Application to Echo Cancellation", Ph. D. dissertation, Dep. Elect. Eng. Univ. of Kent. at Canterbury, U.K., Jan. 1989.

[4] Johnson C. R.," Adaptive IIR Filtering: Current Results and Open Issues", IEEE Trans. on Inform. Theory, vol. IT-30, pp. 237-250, Mar. 1984.

[5] Soleit E. A. and Horne E.," Echo Cancellation using a modified LMS Hyperstable Adaptive Recursive Digital Filter, IERE 5-th Inter. Conf. on Digital Processing of Signals in Communications, Loughborough, pp-231-237. Sept. 1988.

[6] Larimore M.. Trichler J. and Johnson C., "SHARF: An Algorithm for Adapting IIR digital Filters", IEEE Trans. on Acoust. Speech and Signal Processing, vol. ASSP-28, pp. 428-440, Aug. 1980.

[7] Johnson C., "A Convergence Proof for A Hyperstable Adaptive Recursive Filter", IEE Trans. on Inform. Theory, vol. IT-25, pp. 745-749, Nov. 1979. 
\begin{tabular}{|l|l|}
\hline CM-1 & 436 \\
\hline
\end{tabular}

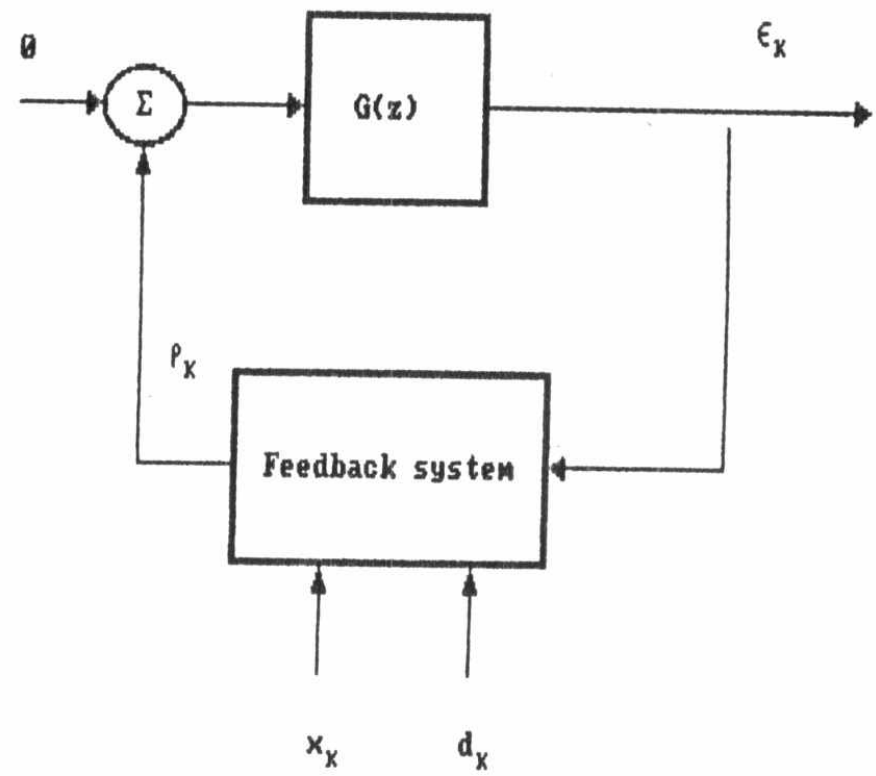

Fig.1 The unforced feedback system

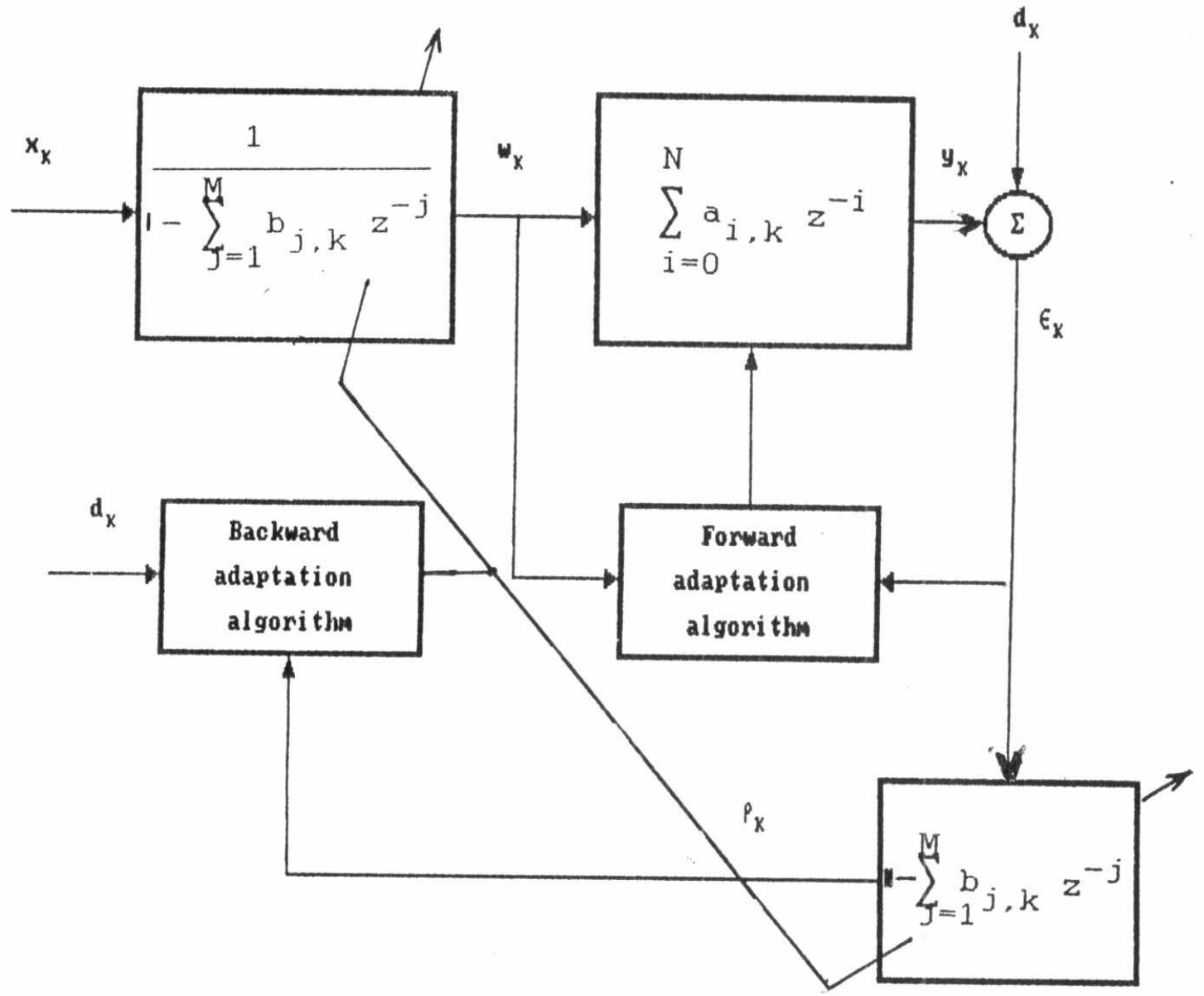

Fig.2 A unimodal adaptive recursive filter 


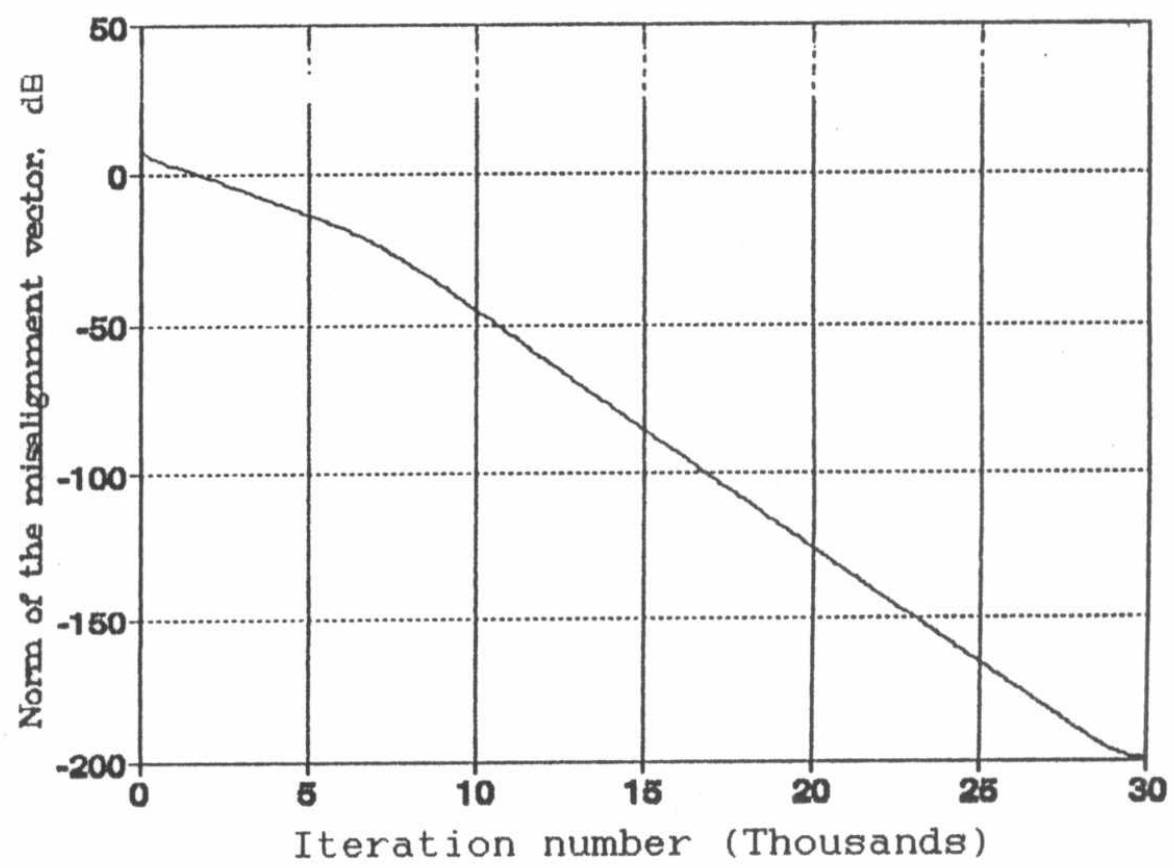

Fig. 3 The learning curve of the adaptive recursive filter

Table 1 The forwand and the backwand coefficients of the adaptive recursive identifier af ter 30000 iterations

\begin{tabular}{|c|c|c|}
\hline Coefficient & Model & Identifier \\
\hline$a_{0}$ & 1.0 & 0.999999 \\
$a_{1}$ & -1.8 & -1.8 \\
$a_{2}$ & 1.1 & 1.1 \\
$b_{1}$ & 1.2 & 1.2 \\
$b_{2}$ & -0.6 & -0.59999 \\
\hline
\end{tabular}

